\title{
Breast Scintigraphy with Breast-Specific $\gamma$-Camera in the Detection of Ductal Carcinoma In Situ: A Correlation with Mammography and Histologic Subtype
}

\author{
Angela Spanu ${ }^{1}$, Daniela Sanna ${ }^{1}$, Francesca Chessa $^{1},{\text { Pierina } \text { Cottu }^{2}, \text { Alessandra Manca }^{3} \text {, and Giuseppe Madeddu }}^{1}$ \\ ${ }^{I}$ Unit of Nuclear Medicine, University of Sassari, Sassari, Italy; ${ }^{2}$ Unit of Surgery, University of Sassari, Sassari, Italy; \\ and ${ }^{3}$ Unit of Pathology, University of Sassari, Sassari, Italy
}

Ductal carcinoma in situ (DCIS) is a subtype of breast cancer encountered increasingly in clinical practice because of the widespread use of screening mammography. In the present study, we evaluated the usefulness of breast-specific $\gamma$-camera (BSGC) scintigraphy in DCIS identification, describing the scintigraphic findings and their correlation with mammography and histologic subtype. Methods: Thirty-three women, aged 41-81 y, with surgically proven DCIS were retrospectively reviewed. Before surgery, all patients underwent breast scintigraphy using a high-resolution semiconductor-based BSGC, starting $10 \mathrm{~min}$ after intravenous injection of $740 \mathrm{MBq}$ of $99 \mathrm{~m}$ Tc-tetrofosmin. All patients had previously undergone mammography. A definitive histologic diagnosis was obtained in all cases after scintigraphy, and the scintigraphic findings were correlated with mammography and histologic subtype. Results: Mammography was positive in 30 of 33 patients (sensitivity, 90.9\%), showing calcifications in 22 of 30 $(73.3 \%)$, masses in 3 of 30 (10\%), and masses plus calcifications in the remaining 5 of $30(16.7 \%)$. Scintigraphy was positive in 31 of 33 patients (sensitivity, $93.9 \%$ ), showing patchy irregular uptake in patients with calcifications and focal uptake in masses; sensitivity was higher in low- to intermediate-grade DCIS than in intermediate/high- and high-grade DCIS $100 \%$ vs. $91.3 \%$ ), but the difference was not statistically significant. Two comedo-type DCIS (one 20-mm intermediate/high-grade and one 15-mm high-grade) with heterogeneously or highly dense breasts at mammography and one papillary low/intermediate-grade DCIS associated with Paget disease were true positive only at scintigraphy. Moreover, scintigraphy better assessed disease extent than did mammography in 5 additional patients. Two comedo-type DCIS (one 6-mm intermediate/highgrade and one 15- $\mathrm{mm}$ high-grade) were true positive only at mammography. The difference in sensitivity between scintigraphy and mammography was not statistically significant. The combined use of mammography and scintigraphy achieved $100 \%$ sensitivity. Conclusion: BSGC scintigraphy proved to be a highly sensitive diagnostic tool in the detection of DCIS, irrespective of histologic subtype, and with a scintigraphic pattern of uptake that correlated well with mammography findings. In our series, BSGC scintigraphy demonstrated

Received Jan. 11, 2012; revision accepted May 21, 2012.

For correspondence or reprints contact: Angela Spanu, Unit of Nuclear Medicine, University of Sassari, Viale San Pietro, 8, 07100 Sassari, Italy.

E-mail: angela.spanu@email.it

Published online Aug. 21, 2012

COPYRIGHT (C) 2012 by the Society of Nuclear Medicine and Molecular Imaging, Inc. a slightly higher sensitivity than mammography and a better assessment of local disease extent. Thus, BSGC scintigraphy should represent a useful adjunctive tool in breast cancer diagnosis.

Key Words: DCIS; breast-specific $\gamma$-camera scintigraphy; mammography

J Nucl Med 2012; 53:1528-1533

DOI: 10.2967/jnumed.112.103010

D

uctal carcinoma in situ (DCIS) of the breast is a noninvasive subtype of primary breast cancer characterized by a malignant proliferation of ductal epithelium with no histologic evidence of invasion through the ductal basement membrane (1). The mortality associated with a pure DCIS without invasion is thus extremely low, generally lower than $1 \%-2 \%$ (2).

However, different behaviors have been observed after the initial treatment; some DCISs remain indolent or are associated with a low risk of developing into invasive cancer, whereas others show a higher tendency to progress to invasive breast cancer or to recur (3). Published reports have shown that rates of recurrence after treatment for pure DCIS range from $5 \%$ to $20 \%$, and approximately $50 \%$ of these are invasive $(4,5)$.

Several studies have been performed to address the malignant potential of DCIS, and it has emerged that into the common definition of DCIS are grouped several lesions with different histopathologic characteristics. Among these, architectural pattern (comedo, cribriform, or papillary), nuclear grade (high, intermediate, or low) and presence of central necrosis (cell death) seem to represent the most important predictors of DCIS aggressiveness, with high nuclear grade and comedo-type DCIS representing the histologic subtypes with the worst prognosis (6).

With the widespread use of screening mammography, the detection rate of DCIS has dramatically increased, currently accounting for approximately $20 \%-30 \%$ of all newly diagnosed breast carcinomas (7), and calcifications, which generally reflect the presence of necrosis, represent the most frequent mammographic finding (8). 
Breast scintigraphy acquired with a breast-specific $\gamma$-camera (BSGC) is a relatively new radioisotopic imaging procedure that is emerging as a useful complementary tool to mammography in the diagnosis of primary breast cancer, especially in dense breasts and in multifocal or multicentric disease (9).

Preliminary studies have demonstrated that BSGC scintigraphy may also be useful in the detection of DCIS, with sensitivity ranging from $87.5 \%$ to $92.3 \%(10-12)$.

In the present study, we further investigated the usefulness of BSGC scintigraphy in the detection of DCIS and correlated scintigraphic features with mammographic findings and histopathologic subtypes.

\section{MATERIALS AND METHODS}

The present study was a retrospective review of a consecutive series of 33 women with newly diagnosed pure DCIS, aged 41-81 y (mean age, $56.8 \mathrm{y}$ ), who underwent BSGC scintigraphy before surgery during January 2006 to June 2011. All investigations were performed in a university hospital setting as part of the clinical care of breast cancer patients. All patients had undergone both clinical examination and mammography before scintigraphy. The clinical examination had been performed in all cases by an experienced surgeon who noted any palpable lump, skin thickening or retraction, and nipple discharge or retraction. Mammographic study included routine craniocaudal and mediolateral oblique views of the breasts; at least one other projection or magnification over the area of suspected lesions was also obtained in all patients. The lesions were described according to the lexicon of the American College of Radiology Breast Imaging Reporting and Data System (13). All 33 patients underwent surgery within 2 wk of scintigraphy. Twenty-one of the 33 were treated with wide-excision breast-conserving surgery, whereas the remaining 12 were treated with mastectomy. Tumor size (in millimeters), the predominant architectural pattern (comedo, cribriform, papillary, micropapillary, or solid), and the nuclear grade (high, intermediate, or low) was assessed in each patient. Nine patients had low- to intermediate-grade DCIS, whereas 24 had mixed intermediate/highor high-grade DCIS. Mean tumor size was $14.3 \mathrm{~mm}$ (range, 0.5-30 $\mathrm{mm})$. Scintigraphic features were correlated with mammographic findings and histopathologic subtypes in all cases. Patients with DCIS associated with a microinvasion component and those with DCIS ascertained at excisional biopsy performed before scintigraphy were excluded from this study.

This retrospective study was performed in accordance with the regulations of the Institutional Review Board. Written informed consent was obtained from all patients. All patient data were treated in accordance with the local privacy rule regulations.

\section{BSGC Scintigraphy}

BSGC scintigraphy was performed starting $10 \mathrm{~min}$ after the intravenous injection of $740 \mathrm{MBq}$ of ${ }^{99 \mathrm{~m}} \mathrm{Tc}$-tetrofosmin (Myoview; Amersham Health-GE Healthcare) in the arm contralateral to the affected breast. Radiolabeling and quality control procedures for the radiotracer were performed according to the manufacturer's instructions. Labeling efficiency was always over $95 \%$. Scintigraphic images were acquired at the technetium peak $(140 \mathrm{keV})$ with a $\pm 10 \%$ energy window using a high-resolution dedicated breast camera (LumaGEM 3200S/12k; Gamma Medica Ideas Inc.) consisting of a small-field-of-view $(20 \times 15 \mathrm{~cm})$ high- resolution, solid-state semiconductor (cadmium zinc telluride) detector mounted on a modified mammographic unit, replacing the radiographic Bucky unit. The camera head was composed of a pixelated $(12,288$ pixels) array of cadmium zinc telluride (pixel size, $1.5 \times 1.5 \times$ $5 \mathrm{~mm}$ ) coupled to an array of amplifiers, the signals from which were conveyed to an electronics readout board. The system was equipped with a high-sensitivity parallel-hole low-energy all-purpose long-bore collimator (hexagonal holes $25.4 \mathrm{~mm}$ long and $2 \mathrm{~mm}$ in diameter, with a septal thickness of $0.3 \mathrm{~mm}$ ) matched to the cadmium zinc telluride elements. The intrinsic spatial resolution was $1.6 \mathrm{~mm}$, and the energy resolution was less than 5\% (average, $4.6 \%$ at $140 \mathrm{keV}$ ).

In all patients, craniocaudal and mediolateral oblique projections (600 s per view) were acquired using a $128 \times 128$ matrix, with the breast positioned between the detector and the compression paddle of the mammographic unit to ensure light compression of the breast parenchyma, reducing its thickness, limiting movement artifacts, and improving lesion contrast. Additional breast projections could be acquired when necessary (e.g., breast larger than the field of view or areas of increased uptake at the border of the field of view or not close to the camera), given the flexibility of the mammographic gantry in breast positioning.

All patients gave their informed consent before undergoing scintigraphy.

\section{Statistical Analysis}

The McNemar test was used to calculate the difference in sensitivity between scintigraphy and mammography. The $\chi^{2}$ test was used to assess statistical differences in the sensitivity of breast scintigraphy among the different histologic subtypes (low- to intermediate-grade DCIS vs. intermediate/high- and high-grade DCIS). The results were considered significant when the $P$ value was less than 0.05 .

\section{RESULTS}

The positivity of both mammography and BSGC scintigraphy in the detection of DCIS in relationship to patient demographics and histopathologic characteristics is reported in Table 1. Both procedures were positive in 28 patients, only scintigraphy was positive in 3 patients, and only mammography was positive in 2 patients. Thus, mammography was positive in 30 of 33 patients (overall sensitivity, 90.9\%), showing calcifications alone in 22 of 30 (73.3\%), a mass alone in 3 of $30(10 \%)$, and a mass plus calcifications in the remaining 5 of $30(16.7 \%)$. Mammography was false-negative in the remaining 3 of 33 patients. Two of these 3 patients, both with heterogeneously or highly dense breasts, had one palpable comedo-type DCIS each (20 and $23 \mathrm{~mm}$ in size), of intermediate/high grade in one and of high grade in the other. The remaining patient had an extensively spreading low/intermediate-grade DCIS in association with Paget disease.

BSGC scintigraphy was true-positive in 31 of 33 patients (overall sensitivity, 93.9\%), including the 3 patients false-negative at mammography, showing a high focal uptake at the level of the palpable lesions in the 2 patients with DCS. In the third patient false-negative at mammography with DCIS and Paget disease, scintigraphy showed a focal area of increased uptake in the areolar region and an irregular area of increased uptake in the surrounding tissue. In the remaining 28 patients 
TABLE 1

Mammography and BSGC Scintigraphy Positivity Related to Patient Characteristics

\begin{tabular}{|c|c|c|c|}
\hline No. of patients & Characteristic & $\begin{array}{l}\text { No. of patients positive } \\
\text { at mammography }\end{array}$ & $\begin{array}{l}\text { No. of patients positive } \\
\text { at BSGC scintigraphy }\end{array}$ \\
\hline & Age at diagnosis $(\mathrm{y})$ & & \\
\hline 10 & $40-50$ & 9 & 8 \\
\hline 15 & $51-60$ & 13 & 15 \\
\hline 5 & $61-70$ & 5 & 5 \\
\hline 2 & $71-80$ & 2 & 2 \\
\hline \multirow[t]{2}{*}{1} & $>80$ & 1 & 1 \\
\hline & Menopausal status & & \\
\hline 11 & Premenopausal & 10 & 10 \\
\hline \multirow[t]{2}{*}{22} & Postmenopausal & 20 & 21 \\
\hline & Physical examination & & \\
\hline 18 & Negative & 18 & 17 \\
\hline 14 & Palpable mass & 12 & 13 \\
\hline \multirow[t]{2}{*}{1} & Nipple discharge & 0 & 1 \\
\hline & Tumor size & & \\
\hline 17 & $\leq 10 \mathrm{~mm}$ & 17 & 16 \\
\hline \multirow[t]{2}{*}{16} & $>10 \mathrm{~mm}$ & 13 & 15 \\
\hline & Vuclear grade & & \\
\hline 2 & Low & 2 & 2 \\
\hline 2 & Low/intermediate & 1 & 2 \\
\hline 5 & Intermediate & 5 & 5 \\
\hline 11 & Intermediate/high & 10 & 10 \\
\hline 13 & High & 12 & 12 \\
\hline
\end{tabular}

positive at scintigraphy, the uptake pattern was focal with round contours in patients with masses at mammography (Fig. 1), focal with irregular contours in patients with distortions, and scattered patchy, irregular, or linear in patients with microcalcifications, varying according to their distribution (Fig. 2). However, in 5 patients true-positive at scintigraphy with only microcalcifications at mammography, radiotracer uptake was more extended than microcalcifications, also involving the areas surrounding them (Fig. 3) and better correlating with surgical findings.

Scintigraphy was false-negative in the remaining 2 of 33 patients, both with comedo-type DCIS (high/intermediategrade in one and high-grade in the other, 6 and $15 \mathrm{~mm}$ in size, respectively) positive at mammography that showed a pattern of pleomorphic calcifications.

Scintigraphy sensitivity was higher in the group of 9 patients with low- to intermediate-grade DCIS than in the group of 24 patients with mixed intermediate/high- and high-grade DCIS (100 vs. 91.3\%), but the difference was not statistically significant $(P>0.05)$.

When scintigraphy sensitivity and mammography sensitivity were mutually compared, the difference was not statistically significant $(P>0.05)$. The combined use of these 2 procedures achieved $100 \%$ sensitivity.

\section{DISCUSSION}

At present, mammography represents the imaging procedure of reference in the identification of DCIS because of the high sensitivity demonstrated in women undergoing screening programs (7), markedly contributing to the in- crease in the detection rate of DCIS over the last 2 decades and offering the opportunity of early diagnosis and prompt treatment.

Currently, most cases of newly diagnosed DCIS are asymptomatic, with only microcalcifications as suggestive mammographic findings. The morphologic features of calcifications encountered in DCIS are extremely variable, although poorly differentiated DCIS generally presents as pleomorphic, linear, branching, and coarse granular microcalcifications, whereas well-differentiated DCIS generally presents as fine granular calcifications or a single cluster of coarse granular calcifications $(14,15)$. However, considerable overlap between the mammographic appearance of microcalcifications and the histopathologic grade of DCIS was observed by several authors $(8,14)$, thus suggesting that the predominant histologic subtype cannot be predicted on the basis of the microcalcification type with a high degree of accuracy.

Approximately $10 \%-20 \%$ of DCIS appears as a mass and not as a calcified lesion; in addition, some cases of DCIS are occult at mammography. Thus, additional imaging modalities besides mammography could be useful for the detection of DCIS.

Breast scintigraphy acquired with a BSGC is emerging as a useful complementary tool to mammography in the diagnosis of primary breast cancer, and it has been proved that this radioisotopic procedure is able to overcome the main disadvantages of mammography, such as low specificity and low sensitivity in dense breasts and in multifocal or multicentric disease (9). Preliminary studies have demonstrated that BSGC scintigraphy may also be useful in the 

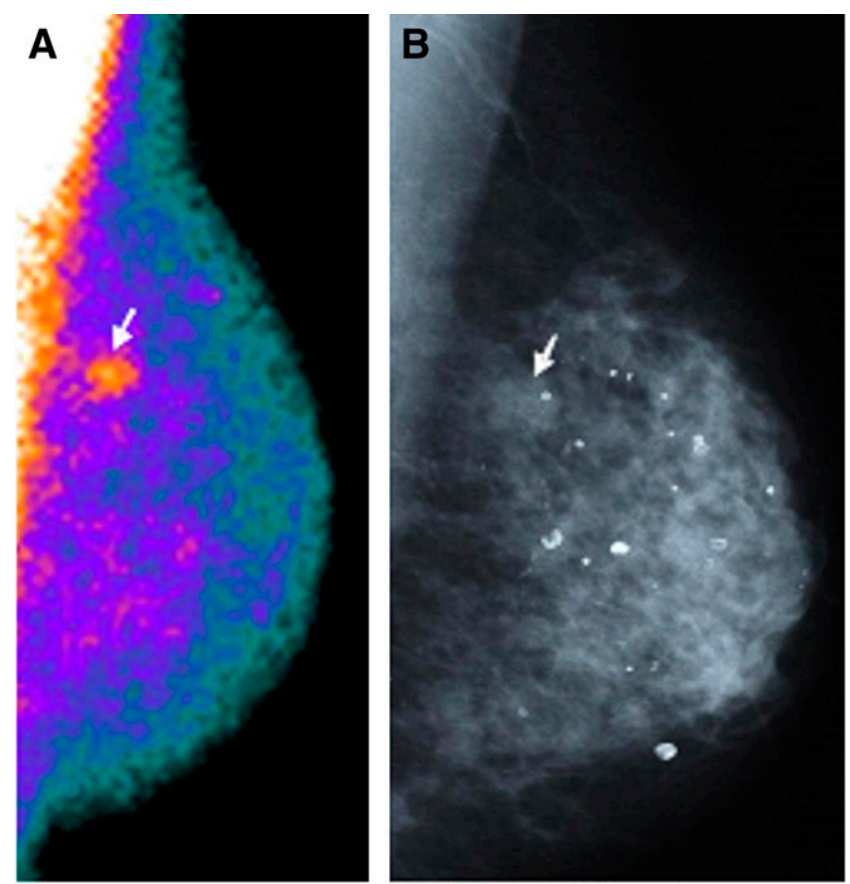

FIGURE 1. A 75-y-old patient with intermediate-grade papillarytype DCIS $(0.8 \mathrm{~cm})$ in external upper quadrant of left breast (arrow) true-positive at scintigraphy $(A)$ as focal area of increased uptake at level of mass evidenced at screening mammography (B).

detection of DCIS, with sensitivity similar to or even higher than that of mammography, in comparative studies $(10,11)$. In a series of 13 patients with 13 surgically proven DCISs, scintigraphy and mammography showed the same sensitivity (92.3\%), with 1 DCIS evidenced only at scintigraphy and 1 DCIS ascertained only at mammography (10). In another series of 20 patients with 22 biopsy-proven DCISs, $91 \%$ of lesions were detected by BSGC scintigraphy and $82 \%$ by mammography, with 2 DCISs evidenced only at BSGC (11).

In the present study performed on a consecutive series of 33 patients with pure DCIS, we further evaluated the usefulness of BSGC scintigraphy in the detection of DCIS and correlated the scintigraphic features with mammographic findings and histopathologic subtype.

Most patients of our series were asymptomatic and had only microcalcifications at mammography; the most frequent microcalcifications were pleomorphic (present in 5 of 9 patients [55\%] with low- to intermediate-grade DCIS and in 14 of 24 patients [58.3\%] with mixed intermediate/ high- and high-grade DCIS). This finding confirms that the histologic grade of DCIS cannot be established on the basis of the mammographic appearance of microcalcifications.

In accordance with previous studies $(10,11)$, BSGC scintigraphy in our series demonstrated a high sensitivity in the detection of DCIS. The fact that false-negative findings occurred in only 2 patients is not easy to explain. Lesion size should be excluded as a limiting factor, since both lesions overcame the spatial resolution of the device. Nor would the histologic subtype or the tumor grade seem to be
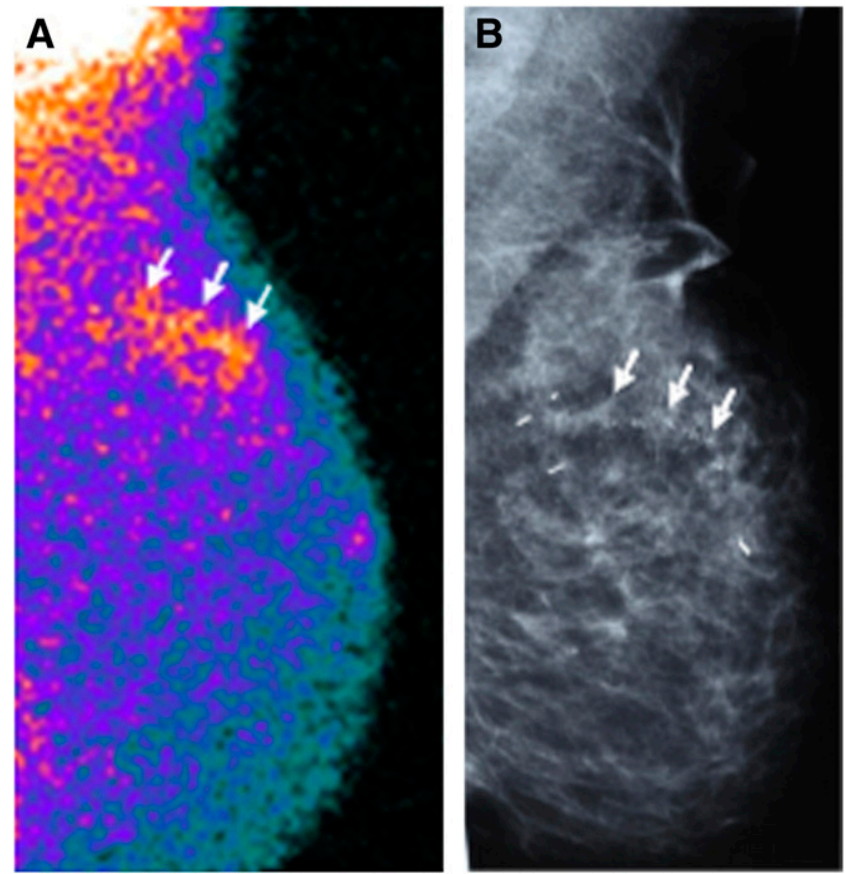

FIGURE 2. A 52-y-old patient with high-grade comedo-type DCIS in external upper quadrant of left breast (arrows) true-positive at scintigraphy (A) as scattered irregular increased uptake matching distribution of microcalcifications evidenced at screening mammography $(\mathrm{B})$.

responsible for these false-negative findings, since all other cases of mixed intermediate/high- and high-grade comedotype DCIS were identified by scintigraphy. In addition, no statistically significant difference in sensitivity was found for scintigraphy when low- to intermediate-grade DCIS was compared with intermediate/high- and high-grade DCIS.

Moreover, in the current study, scintigraphy proved more sensitive than mammography, with 3 cases of DCIS evidenced only at scintigraphy. However, 2 further cases of DCIS were detected only at mammography, thus suggesting the usefulness of the combined use of both procedures, by which $100 \%$ of tumors was identified. Among the 3 cases of DCIS missed at mammography and evidenced at scintigraphy, there was an extensively spreading low/intermediate-grade DCIS underlying the nippleareolar region affected by Paget disease. Paget disease is a rare malignancy associated with an invasive or in situ carcinoma in $87 \%-100 \%$ of cases, even in the absence of a palpable mass or mammography findings (16). The scintigraphic evidence of concomitant DCIS, as in the patient included in our series, seems to suggest that breast scintigraphy may be used as an adjunctive diagnostic tool for more accurate staging of patients with Paget disease, guiding the surgeon in planning the most appropriate surgical treatment.

Furthermore, we observed a high correlation between scintigraphic uptake pattern and mammography findings, the uptake being focal in patients with masses at mammography but irregular, linear, or patchy and scattered in patients with 

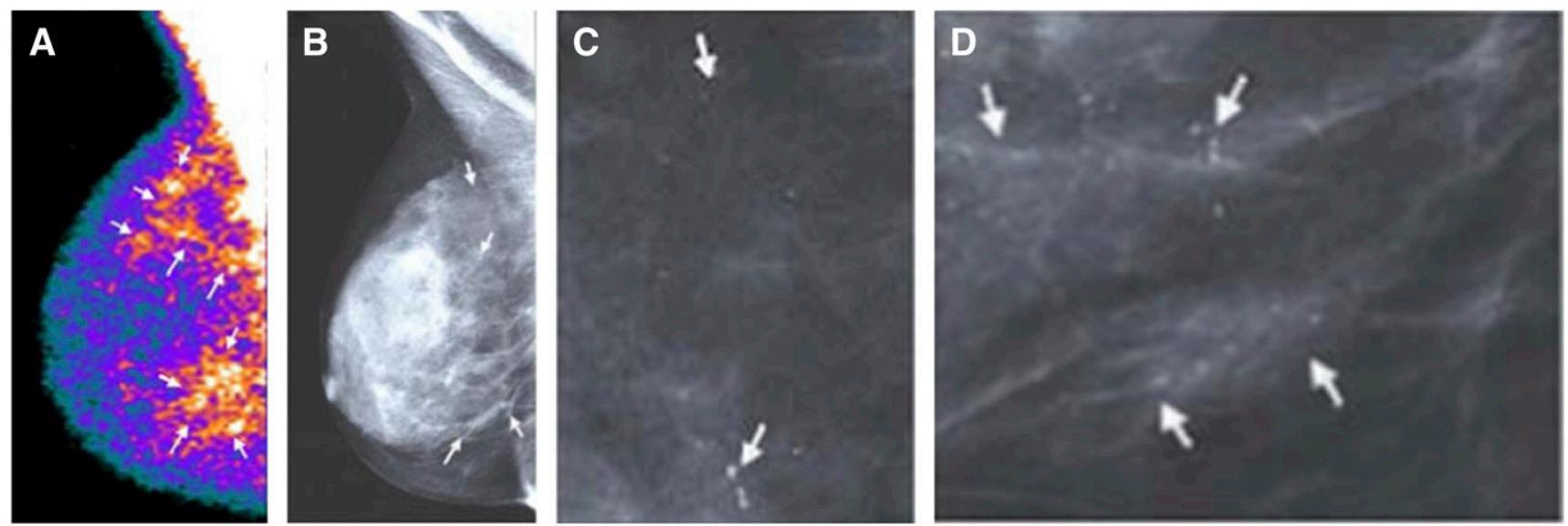

FIGURE 3. A 59-y-old patient with high-grade comedo-type DCIS. Breast scintigraphy (A) showed multiple foci of increased uptake scattered in both upper and lower external quadrants of right breast (arrows) where pleomorphic calcifications (arrows) were evidenced at screening mammography (B). Spot magnification mammograms that better illustrate microcalcifications in upper (C) and lower (D) external quadrants are also shown. Scintigraphy surpasses mammography in demonstrating greater disease extent, especially in upper external quadrant, where radiotracer uptake was more extended than microcalcifications, better correlating with surgical findings that evidenced multiple disseminated tumor foci.

microcalcifications, and matching the distribution of the microcalcifications. Moreover, breast scintigraphy gave more accurate local disease staging than mammography in some patients in whom microcalcifications underestimated tumor extent ascertained at surgery.

The limitation of mammography in the preoperative assessment of DCIS extent is well recognized since microcalcifications may coexist in the same DCIS lesion with uncalcified areas not associated with pathologic findings at mammography $(17,18)$. Thus, in the era of breast-conserving surgery, there could be the risk that mammographically undetected areas would not be surgically removed, thus increasing the rate of surgical reoperation and local recurrences.

On the other hand, the superiority of BSGC scintigraphy with respect to mammography in the preoperative assessment of breast cancer extent has been already been demonstrated in a large series of patients with invasive breast cancer, in some of whom only scintigraphy evidenced additional small invasive foci or DCIS around the index tumor $(9,10)$. However, further studies in larger series of patients are needed to prospectively assess the performance of BSGC scintigraphy in the preoperative evaluation of disease extent in patients with the diagnosis of DCIS and to clarify whether the additional use of BSGC may allow more selective and accurate surgical approaches to removing DCIS, thus reducing local recurrences and improving patient outcome.

The major limitation of the present study was the relatively small number of patients, although our series was larger than the casuistries previously reported in the literature $(10,11)$. Furthermore, although sample size limits comparative analysis, to our knowledge the present study is the first reported in the literature that correlates scintigraphic findings with mammographic patterns and histologic subtypes. Further studies with a larger number of patients will be needed to confirm our data.

\section{CONCLUSION}

Breast scintigraphy acquired with a BSGC proved a highly sensitive diagnostic tool in DCIS detection, irrespective of the histopathologic subtype, and with a scintigraphic uptake pattern correlating well with mammography findings. In our series, BSGC scintigraphy demonstrated a higher sensitivity than mammography and a better assessment of local disease extent. A wider clinical application of BSGC scintigraphy is thus suggested as an adjunctive diagnostic tool to mammography in the diagnosis of primary breast cancer, even in asymptomatic patients with only microcalcifications found at mammography.

\section{DISCLOSURE STATEMENT}

The costs of publication of this article were defrayed in part by the payment of page charges. Therefore, and solely to indicate this fact, this article is hereby marked "advertisement" in accordance with 18 USC section 1734.

\section{ACKNOWLEDGMENT}

This study was funded in part by a grant from the "Fondazione Banco di Sardegna." No other potential conflict of interest relevant to this article was reported.

\section{REFERENCES}

1. Burstein HJ, Polyak K, Wong JS, Lester SC, Kaelin CM. Ductal carcinoma in situ of the breast. N Engl J Med. 2004;350:1430-1441.

2. Allegra CJ, Aberle DR, Ganschow P, et al. National Institutes of Health State-ofthe-Science Conference statement: diagnosis and management of ductal carcinoma in situ September 22-24, 2009. J Natl Cancer Inst. 2010;102: $161-169$.

3. Roses RE, Arun BK, Lari SA, et al. Ductal carcinoma-in-situ of the breast with subsequent distant metastasis and death. Ann Surg Oncol. 2011;18:28732878.

4. Fisher B, Land S, Mamounas E, Dignam J, Fisher ER, Wolmark N. Prevention of invasive breast cancer in women with ductal carcinoma in situ: an update of the 
National Surgical Adjuvant Breast and Bowel Project experience. Semin Oncol. 2001;28:400-418.

5. Bijker N, Meijnen P, Peterse JL, et al. Breast-conserving treatment with or without radiotherapy in ductal carcinoma-in-situ: ten year results of European Organization for Research and Treatment of Cancer randomized phase III trial 10853 - a study by the EORTC Breast Cancer Cooperative Group and EORTC Radiotherapy Group. J Clin Oncol. 2006;24:3381-3387.

6. Lari SA, Kuerer HM. Biologic markers in DCIS and risk of breast recurrence: a systematic review. J Cancer. 2011;2:232-261.

7. Ernster VL, Ballard-Barbash R, Barlow WE, et al. Detection of ductal carcinoma in situ in women undergoing screening mammography. J Natl Cancer Inst. 2002;94:1546-1554.

8. Slanetz PJ, Giardino AA, Oyama T, et al. Mammographic appearance of ductal carcinoma in situ does not reliably predict histologic subtype. Breast J. 2001; 7: $417-421$.

9. Spanu A, Chessa F, Meloni GB, et al. The role of planar scintimammography with high-resolution dedicated breast camera in the diagnosis of primary breast cancer. Clin Nucl Med. 2008;33:739-742.

10. Spanu A, Chessa F, Meloni GB, et al. Scintimammography with high resolution dedicated breast camera and mammography in multifocal, multicentric and bilateral breast cancer detection: a comparative study. Q J Nucl Med Mol Imaging. 2009;53:133-143.
11. Brem RF, Fishman A, Rapelyea JA. Detection of ductal carcinoma in situ with mammography, breast specific gamma imaging, and magnetic resonance imaging: a comparative study. Acad Radiol. 2007;14:945-950.

12. Keto JL, Kirstein L, Sanchez DP, et al. MRI versus breast-specific gamma imaging (BSGI) in newly diagnosed ductal cell carcinoma-in-situ: a prospective head-to-head trial. Ann Surg Oncol. 2012;19:249-252.

13. D'Orsi CJ, Kopans DB. Mammographic features analysis. Semin Roentgenol. 1993;18:204-230.

14. Stomper PC, Connolly JL. Ductal carcinoma in situ of the breast: correlation between mammographic calcification and tumor subtype. AJR. 1992;159:483-485.

15. Holland R, Hendriks JH. Microcalcifications associated with ductal carcinoma in situ: mammographic-pathologic correlation. Semin Diagn Pathol. 1994;11:181192.

16. Sakorafas GH, Blanchard K, Sarr MG, Farley DR. Paget's disease of the breast. Cancer Treat Rev. 2001;27:9-18.

17. Shiraishi A, Kurosaki Y, Maehara T, Suzuki M, Kurosumi M. Extension of ductal carcinoma in situ: histopathological association with MR imaging and mammography. Magn Reson Med Sci. 2003;2:159-163.

18. Menell JH, Morris EA, Dershaw DD, Abramson AF, Brogi E, Liberman L. Determination of the presence and extent of preductal carcinoma in situ by mammography and magnetic resonance imaging. Breast J. 2005;11:382390. 